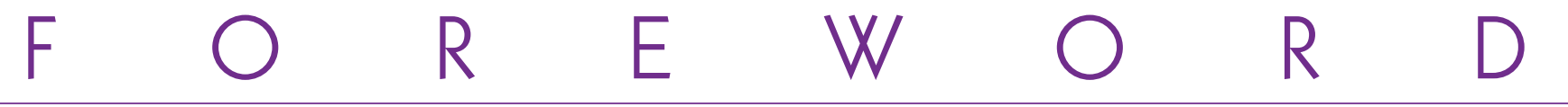

\title{
Medical records and patients with specific needs
}

\author{
D. Brézulier ${ }^{1}$, O. Sorel ${ }^{2}$
}
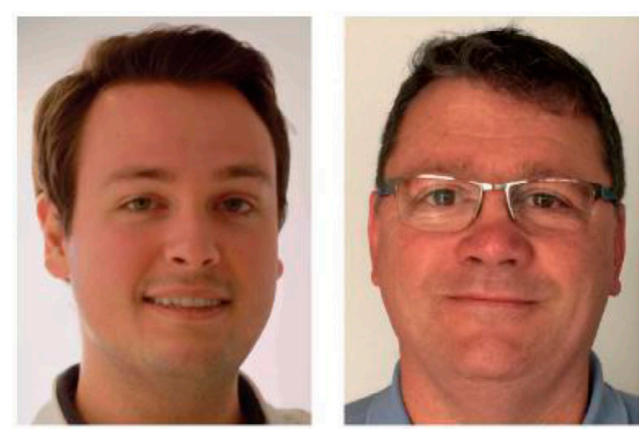

1 Assistant University Hospitalist, Specialist in Orthodontics, CHU [University Hospital Center] Rennes, Université de Rennes 1

2 Professor - Head of the department of Orthodontics, Hospital Practitioner, CHU [University Hospital Center] Rennes, Université de Rennes 1

The philosopher Mark Hunyadi of I'Université Laval defined three complementary and incontrovertible notions in Science and in Medicine:

- caution applies to known risks that we know how frequently they occur;

- prevention, for its part, remedies proven risks whose frequency is not clearly defined;

- precaution applies to risks where neither the magnitude nor the probability of occurrence can be calculated with certainty.

Prudence and precaution can be considered as obstacles to innovation and advances in therapeutics. Besides, is it not a precautionary principle?

This principle, applied to orthodontic care, can be conceived in terms of level of knowledge and requirements with the goal of making our care more secure and efficient.

We then become fully aware of the weight "of knowledge" in the benefit-risk relationship.

However, "knowledge" can no longer be confined to a purely technical field. It is on the basis of this postulate that we chose to dedicate an issue of the Dento-Facial Orthopedic Review to patients with specific needs, who are fortunately few in number and who we are therefore less accustomed to dealing with, but who still require all our vigilance.

In this issue, Yves Soyer proposes setting up the medicolegal framework of patients' medical records, which are very important elements and often the first contact of practitioners with the particularities of patients with specific needs.

Address for correspondence:

Damien Brézulier - 2, Avenue du Professeur Léon Bernard - 35000 Rennes

E-mail: damien.brezulier@univ-rennes1.fr

This is an Open Access article distributed under the terms of the Creative Commons Attribution License (http://creativecommons.org/licenses/by/4.0), which permits unrestricted use, distribution, and reproduction in any medium, provided the original work is properly cited. 
An editorial by Bernard Peckels makes us reflect on what is "good health."

We then mention taking care of patients with special needs, particularly those followed in the field of pediatriconcology, with the article by Émile Boyer.

Then, we discuss patients with endocrinopathies and see the impact these attacks have on growth and skeletal and dental maturation in Fanny Duhamel's article.

We then consider the management of patients with heart disease in the work by Olivier Leborgne.
The interceptive management of infants with Down syndrome is dealt with by Jean-Louis Sixou.

Finally, we focus on the work by Mickael Sebbag and the Bordeaux team on the diagnosis and management of patients suffering from Brodie syndrome.

We conclude this issue by reporting on a case treated by Bertrand Ducroz in the departement of Orthodontics, Rennes Hospital.

We hope that you enjoy reading this issue and that your questions are answered. 